\title{
THE EXPERIMENTAL MICROCLIMATE MONITORING SYSTEM OF THE PAPAL BASILICA OF SAINT FRANCIS OF ASSISI, ITALY
}

\author{
FABIO GARZIA ${ }^{1,2,3,4}$ \& ALESSANDRO CULLA ${ }^{1}$ \\ ${ }^{1}$ Safety \& Security Engineering Group - DICMA, SAPIENZA - University of Rome, Italy \\ ${ }^{2}$ Wessex Institute of Technology, Southampton, UK \\ ${ }^{3}$ European Academy of Sciences and Arts, Salzburg, Austria \\ ${ }^{4}$ Foundation for the Basilica of Saint Francis in Assisi, Assisi, Italy
}

\begin{abstract}
Peculiar, unique and complex cultural heritage sites, such as the Papal Basilica and the Sacred Convent of Saint Francis in Assisi in Italy, need a great effort to ensure visitors security and safety, cultural heritage preservation and protection and great usability for visitors with particular reference to visitors with disabilities and for all the people and personnel normally present for the site management. This goal can be reached using integrated systems and innovative technologies, such as Internet of Everything (IoE), which allows to connect people, things (mobile terminals, smart sensors, devices, actuators, wearable devices, etc.), data/information/knowledge and processes to reach the desired purposes. The IoE system must able to implement and support an integrated multidisciplinary model for security and safety management (IMMSSM) for the specific context, using a multidisciplinary approach. From this point of view, due to the intensive presence of unique frescos and due to the enormous number of visitors that visit every year the whole Papal Basilica, it is necessary to monitor the microclimate conditions to avoid of reaching critical conditions that could activate damaging processes of the precious and unique frescos. The purpose of this paper is to illustrate the experimental microclimate monitoring system of the Papal Basilica of Saint Francis in Assisi, based on suitable microclimate monitoring modules (MCCMs), which represents one of the sub-systems of a proper integrated technological systema framework based on IoE (ITSF-IoE), that is going to be realized subsystem by sub-system, to finally support an efficient IMMSSM capable of ensuring a plenty of functionalities aimed at simplifying the management of such a unique and complex site such as the considered one, not only from security and safety point of view.

Keywords: cultural heritage protection, microclimate monitoring system, security management, safety management, Internet of Things, Internet of Everything, integrated security system, integrated safety system.
\end{abstract}

\section{INTRODUCTION}

The Papal Basilica and the Sacred Convent of Saint Francis in Assisi in Italy represents a unique and peculiar cultural heritage site where the mortal remains of Saint Francis are housed since 1230 A.D.

Millions of pilgrims and visitors coming from all over the world visit this site each year that, from 2000 A.D., together whit other Franciscan sites in the surrounding and the entire Assisi town, have been appointed as World Heritage by UNESCO.

Peculiar, unique and complex cultural heritage sites, such as the considered one, need a significant effort to ensure visitors security and safety, cultural heritage preservation and protection and great usability for visitors with particular care to visitors with disabilities and for all the people and personnel normally present for the site management.

This goal can be reached using integrated systems [1]-[5] and innovative technologies, such as Internet of Everything (IoE), which allows to connect people, things (mobile terminals, smart sensors, device, actuators, wearable devices, etc.), data/information/knowledge and processes to reach the desired goals [6], [7]. 
The IoE system must be able to implement and support an integrated multidisciplinary model for security and safety management (IMMSSM) [8], for the specific, peculiar and unique context, using a multidisciplinary approach [9]-[12].

The IMMSSM can be implemented and supported using a proper Integrated Technological System Framework based on IoE (IoE-ITSF) which allows the full functionalities of the IMMSSM with high flexibility and modularity. In this way, it is possible to translate any eventual modification of the IMMSSM into a fast and low-cost modification of the ITSF at any time, ensuring always the best performances of IMMSSM.

From this point of view, different activities to start to create the different subsystems of a proper IoE-ITSF suitable for the considered site have already started [13]-[14].

Due to the presence of strong architectural vincula, it is necessary to take care in wires and devices installation [15] and, for this reason, a proper laser scanning activity has already started [13], [14], so that all the information will be transferred on a proper BIM (Building Information Modelling) model, to use all its powerful performances, including safety and security management [16].

Due to the intensive presence of unique frescos all over the lower and upper Churches and due to the enormous number of visitors that visit every year the entire Papal Basilica, it is necessary to monitor the microclimate conditions to avoid of reaching critical conditions that could activate damaging processes of the frescos.

In this way, it is possible to relate, in real time, the number of visitors with respect to the microclimate parameters and to act with proper real-time-actions that allow to prevent critical situation for the frescos, within a suitable IMMSSM supported by a proper integrated technological system framework based on IoE (IoE-ITSF).

Further, it is necessary to use low architectonical impact devices, due to the restrictive vincula imposed by the unique internal architecture.

From this point of view, suitable low dimensions micro-controllers have been used, equipped with proper microclimate sensors, studying and realizing an appropriate communication protocol which allows them of working in a quasi-ad-hoc wireless network modality.

Particular care was taken and great efforts were made to reduce the dimensions of the single monitoring module which represents the fundamental flexible element of the monitoring system.

The microcontrollers are endowed by a proper back up electrical energy supply which allows them to work correctly, for a long time, even in absence of main electrical supply.

In this way, it is possible to add, in an effortless way and at any time, any microclimate monitoring module (MCMM) with a quasi-null or totally null architectonical impact inside the Papal Basilica to realize a capillary monitoring system.

The purpose of this paper is to illustrate the experimental microclimate monitoring system of the Papal Basilica of Saint Francis in Assisi, based on suitable MCCMs, which represents one of the sub-systems of a proper ITSF-IoE, that is going to be realized sub-system by sub-system, to finally support an efficient IMMSSM capable of ensuring a plenty of functionalities aimed at simplifying the management of such a unique and complex site such as the considered one, not only from security and safety point of view.

\section{THE PAPAL BASILICA AND THE SACRED CONVENT OF SAINT FRANCIS IN ASSISI}

The Papal Basilica, where unique frescoes by Giotto and other famous painters are present, is composed by three stratified structures:

1. the tomb of Saint Francis, located at the lower level. 
2. The lower Basilica, whose altar is just above the tomb of Saint Francis.

3. The upper Basilica, located above the lower Basilica.

Inside the Sacred Convent are also present a museum, a library and a plenty of places where spiritual and cultural activities are hosted.

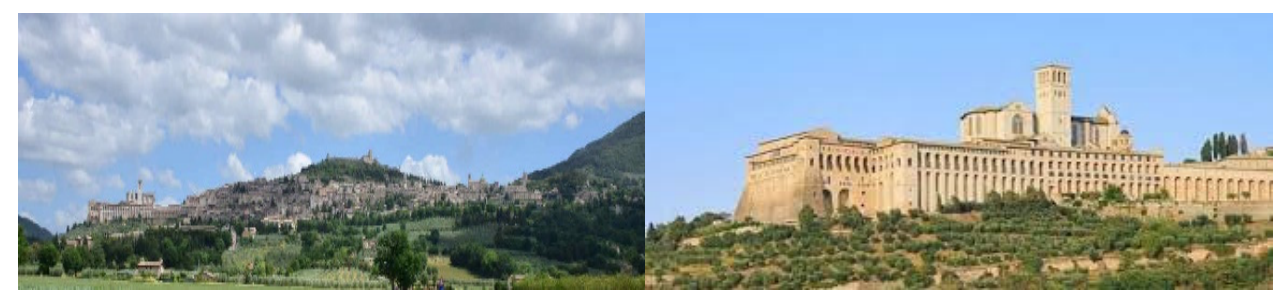

Figure 1: (a) Panoramic view of Assisi where the Sacred Convent is visible on the left; (b) Panoramic view of the Papal Basilica and the Sacred Convent of Saint Francis in Assisi.

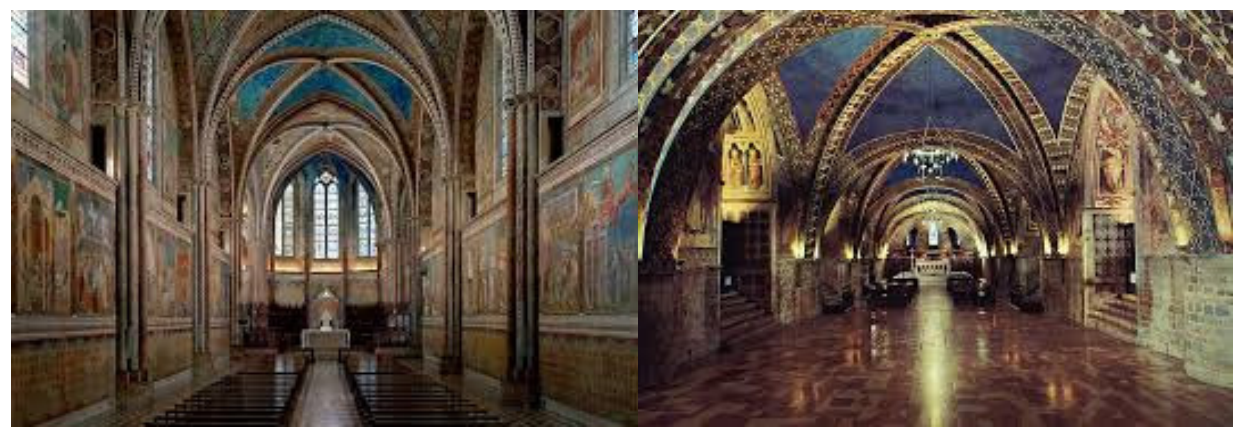

Figure 2: (a) View of the interior of the upper Basilica where the Giotto's frescoes are visible; (b) View of the interior of the lower Basilica.

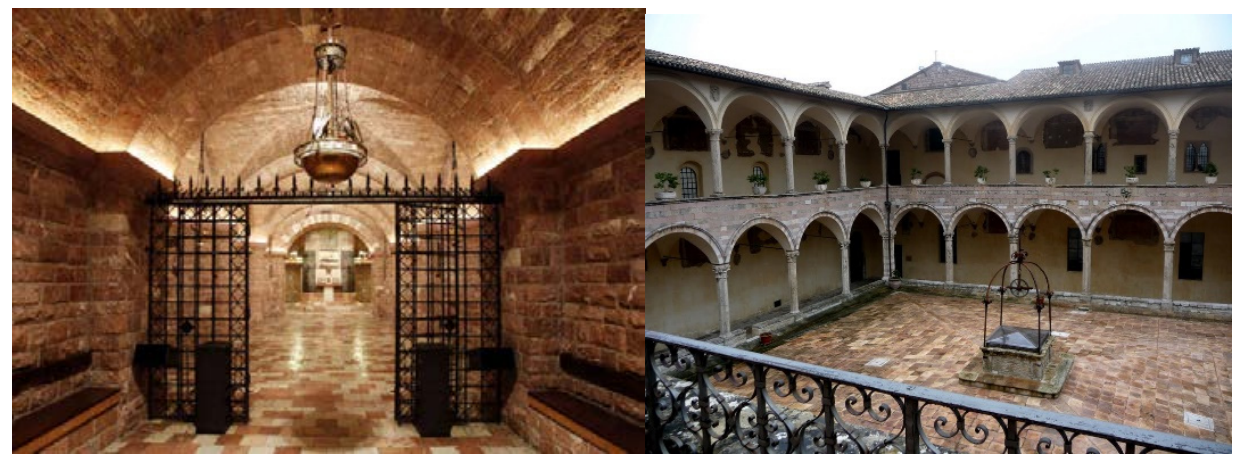

Figure 3: (a) View of the Tomb of Saint Francis; (b) View of the main cloister. 


\section{THE MICROCLIMATE MONITORING SYSTEM}

The monitoring system is based on suitable low dimensions micro-controllers (MCMMs), equipped with proper microclimate sensors, studying and realizing an appropriate communication protocol which allows them of working in a quasi-ad-hoc wireless network modality.

The architecture of the system is shown in Fig. 4.

As it is possible to see from Fig. 4, the MCMMs can use wireless connections, so that it is possible to install them just everywhere, respecting the strong architectural restrictions of the Basilica. They are mainly connected to proper wireless router/gateway (WRG) which communicate with them and adapt their data to send them to the IoE redundant server and to the related database that collects rough data to be later computed according to the requested needs.

Since the system is designed to be modular and reliable, so that it is possible to add, at any time, any further MCMM, the modules communicate with the nearest WRG but they also communicate with the nearest modules so that, in case of malfunctioning of a WRG, the MCMMs forward the data each other to reach the nearest working WRG which can receive the microclimate data and can send them to the IoE server and the related database, ensuring a high reliability to the entire system.

Anyway, in case of severe damage of the communications, the MCMMs can store microclimate data coming from their sensors for a long time, so that, as soon as communications are recovered, they sent all the data stored to the IoE server and wait for the receiving of acknowledge before deleting data from their internal memory.

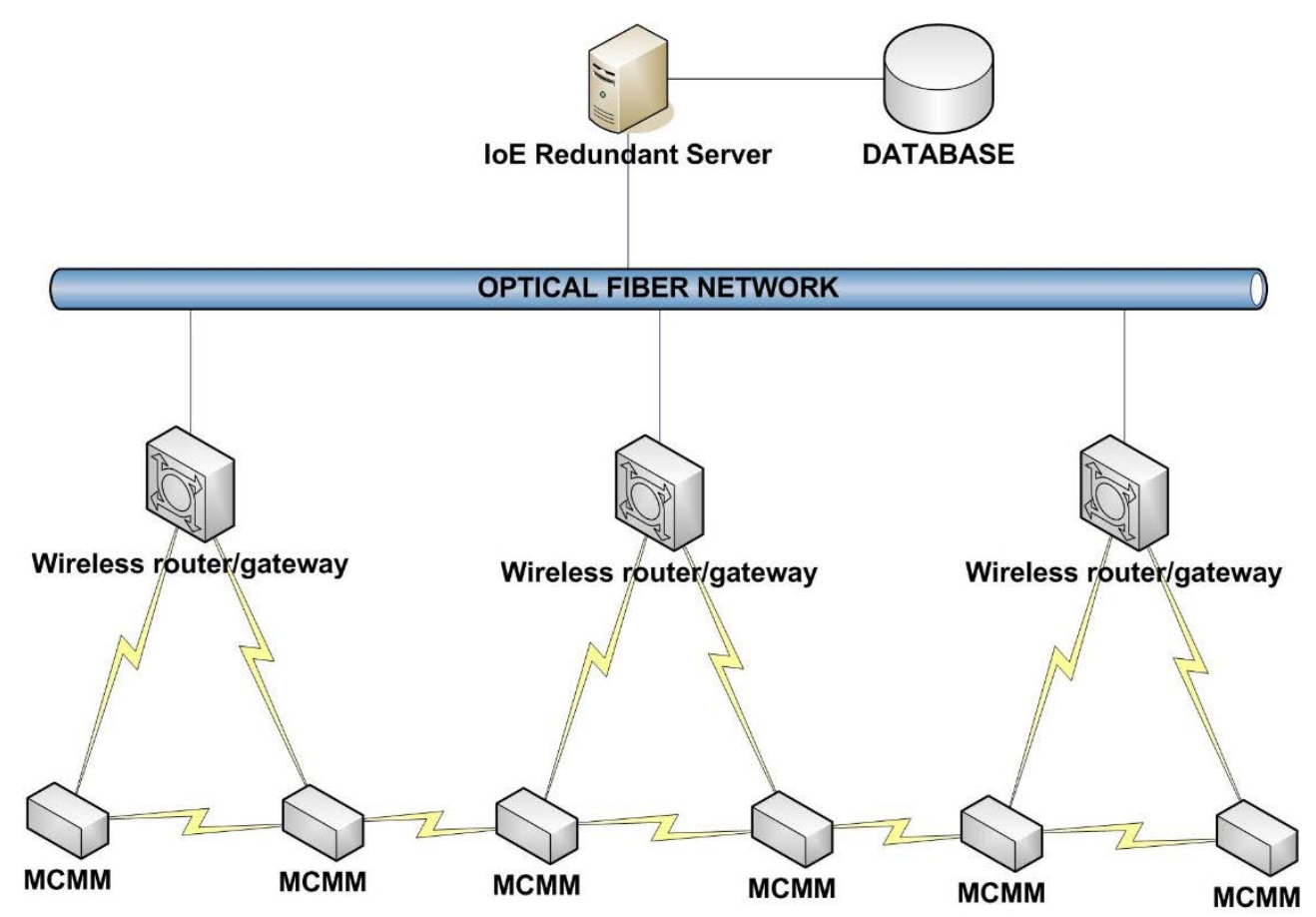

Figure 4: Architecture of the microclimate monitoring system. 
The IoE redundant server is properly connected to the high reliability database, where rough microclimate data are stored, so that is capable to do any kind of data computation according to present and future needs.

Anyway, rough microclimate data are properly stored in a proper external back to ensure a proper backup and related reliability of data storage.

The communication protocol has been studied to reduce as more as possible the band occupation since the MCMM works in the ISM band that is used also by other IoT/IoE devices and services. From this point of view, the MCMM transmits a proper string containing the data, the time and the rough data coming from all sensors at regular interval whose variability can be changed at will, considering the quite slow variability of microclimate parameters involved. Each MCMM checks, before transmitting, the occupation state of the channel to avoid collision with other transmitting MCMMs and if the channel is free, it transmits its data string, waiting for the acknowledge of the correct receiving of it before deleting the data string. If no acknowledge is received, the MCMM start to store the strings in its internal memory, to try to send them later, when the communication channel is available again. In this way, no data is lost, ensuring a high reliability to the entire system.

The MCMM are equipped with different microclimate sensors such as: temperature, relative humidity, carbon dioxide, methane and a plenty of other organic and non-organic gases so that they can constantly monitor the quality of internal microclimate with particular care with respect to the organic substances released by visitors that in some periods of the year can reach a valuable number. The sensors generally produce a voltage variable in quite reduced range as a function of the variability range of the measured parameters. For this reason, proper calibration tests were made to ensure the correct conversion of the values of the measured physical parameters into low voltages. Further, due to the reduced voltage output range of the sensors, particular care was given to the positioning of the components to avoid any interfering between the electronics circuits and the cable/lines that could generate undesired noise, inducing not neglectable errors in the measurement process.

Particular care was taken and great efforts were made to reduce the dimensions of the single monitoring module which represents the fundamental flexible element of the monitoring system on the field. For this reason, the MCMM module have been designed basing on a double layer structure. The lower layer is represented by the microcontroller while the upper layer is represented by the different sensors. In this way, the computing hardware is properly separated from the sensors. The design of the upper layer was properly studied to avoid that the microclimate sensors could interfere each other. Since the most of gas sensors use a catalytic effect, they generate a reduced quantity of heat which, due to the continuous work, can increase the internal temperature of the module. For this reason, the temperature and relative humidity sensors have been properly installed and separated from the other organic gas sensors, so that they measurements are not influenced by the heat produced from them. Further, particular care was given to the box that host the MCMM, as illustrated in the following.

The microcontrollers are endowed by a proper back up electrical energy supply which allows them to work correctly, for a long time, even in absence of main electrical supply. Even if they are characterized by a low energy consumption, the MCMMs have properly programmed to reduce, as more as possible, the energy needs, so that they can operate for a long time using their rechargeable battery. Since the MCMMs could be not installed in comfortable position to be reached for maintenance activity, due to the architectural restrictions of the Basilica that allow to install them only in a reduced number of positions, the compact rechargeable batteries of each MCMM are hosted in a separate box so that it is possible to substitute it in an effortless way in case of malfunctioning, greatly simplifying the 
maintenance operations. Further, it is possible to reduce the dimensions of the entire device, separating the MCMM from the compact rechargeable battery, so that the battery itself can be properly installed at a certain distance from the MCMM, reducing as more as possible the architectural impacts of the devices.

The box that hosts the MCMM has been designed on purpose, since is necessary to allow the sensors to be constantly in contact with air, avoiding any restriction about air flows which must enter the MCMM to be measured by the different sensors. From this point of view, a proper fluid dynamic study was performed, whose validation was made on the field, ensuring that all sensors can operate correctly, providing the right results, without interfering each other. The box was studied and created on purpose using a 3D printer, that allow to choose also the right colour as a function of any installing position, greatly increasing the architectural and aesthetic impact of the module.

Thanks to the flexibility of the MCMM and of the system architecture, it is possible to add, in an effortless way and at any time, any microclimate monitoring module (MCMM) with a quasi-null or totally null architectonical impact inside the Papal Basilica and the entire Sacred Convent to realize a capillary monitoring system.

\section{THE ARTIFICIAL NEURAL NETWORK CORE OF THE SYSTEM}

The system must not only monitor in real time the microclimate but must also be able to forecast the future microclimate as a function of the actual situation, so that it can send this information to the IoE server. In this way, the IoE server can activate all the necessary countermeasures such as reduce the number of visitors, activate the cooling/fanning system of the Tomb so that a part of that air can be used to improve the quality of the air of the lower Church (since they are connected through two separated stairs: one used to let visitors descend into the Tomb and one used to let visitors exit the Tomb), etc.

This kind of early warning or forecasting behaviour can be performed using artificial neural networks (ANNs) [17], [18]. From this point of view, a proper ANN core that can run on the IoE server or any other computing resources has been studied and tested, showing interesting results for the desired goals point of view.

The ANN based core utilizes the input microclimate parameters deriving from different MCMMs positioned in various parts of the Basilica, whose proper combination can reveal the future creation of microclimate critical conditions. In this way, it can operate as a microclimate early warning system.

It is evident that the more the input parameters and the desired outputs and the more the complexity of the ANN. For this reason, a proper quite simple ANN scheme has been designed and tested, so that it can correctly operate without great computational resources.

The early warning ANN is composed by three layers represented by the input layer, the hidden layer and the output layer.

The input layer accepts as input the $\mathrm{N}$ single values of the microclimate parameters coming from the different MCMMS and attempts to forecast the upcoming values of the same parameters. It is therefore composed by $\mathrm{N}$ neurons.

The hidden layer is composed by $\mathrm{H}$ neurons which accept, as inputs, all the outputs of the first layer.

The output layer is composed by $\mathrm{R}$ neurons which accept as inputs all the outputs of the hidden layer. Only a neuron at once of this layer activates and gives an output as a function of the warning or action that must be produced according to the desired goal.

From this point of view, the input layer behaves as a forecaster while the output layer behaves as a classifier. 
The input layer of the ANN must therefore learn the temporal behaviour of a given input parameter that is to predict its future value as a function of the previous values, while the hidden layer, using as inputs the outputs of the input layer, works in a collaborative way with the output layer, aiding the output layer in classifying the different critical situations of microclimate by activating only a chosen neuron per critical situation.

The input layer of the ANN uses the last B values of a given microclimate parameter, which are temporally spaced according to the desired precision, and the input layer must forecast the next value of the given parameter. This means that the input layer must learn to forecast the future value of the given microclimate parameter basing on the previous B values of the same parameter.

The operative scheme of a single neuron of the input layer of the ANN is shown in Fig. 5.

The block elements labelled $\Delta \mathrm{T}$ represent temporal delay elements which give as output their input after a time interval equal $\Delta \mathrm{T}$. The system acts as a predictive filter which estimates the actual value of the input variable once known B previous value of the same variable.

The actual value of the considered parameter, is fed into the system that calculates the forecasted value of the same parameter. The system therefore uses the actual value of the considered parameter and its previous B-1 values.

The forecasting interval can be chosen by introducing a certain number of delays elements in the back loop. In fact, since the back loop is used to calculate the error between the actual value and the predicted value, if input layer is desired to forecast what is the value of the considered parameter $\mathrm{D}$-time intervals $\Delta \mathrm{T}$ in advance, it is necessary to introduce $\mathrm{D}$ delays elements. In this way, the D-th delay element provides at its output the considered forecasted parameter $\mathrm{D}$ time intervals in advance and this variable is desired to be equal to the actual value. The comparison between these two variables is done by the error unit that computes their difference: if the error is different from zero the weight / bias / slope adjuster unit trains the ANN to improve the forecasting ability of the system. If the error is equal to zero, it means that the system can properly forecast the actual value of the considered parameter D time intervals in advance and that it works properly. Each neuron of the first layer of the ANN is trained by adjusting the weights/ bias / slope of the ANN. Since each considered parameter can assume analogic values, because they represent microclimate values, and the learning function is linear, the output of the neuron can assume any value.

The operative scheme of a single neuron of the hidden layer and the output layer of the ANN are shown in Fig. 6 and Fig. 7 respectively.

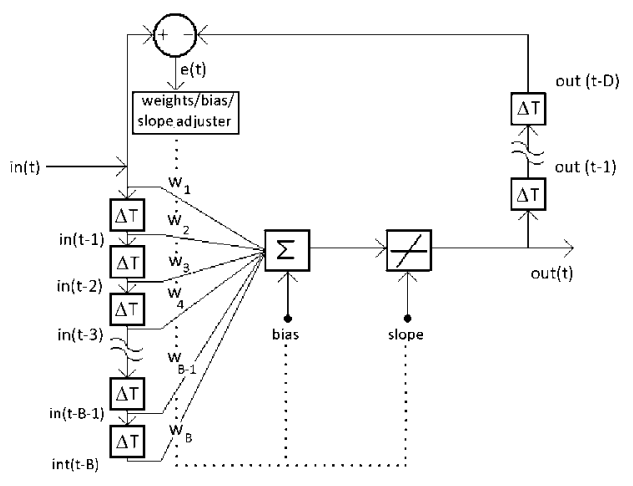

Figure 5: Scheme of a single neuron of the input layer (forecasting layer) of the considered ANN. 


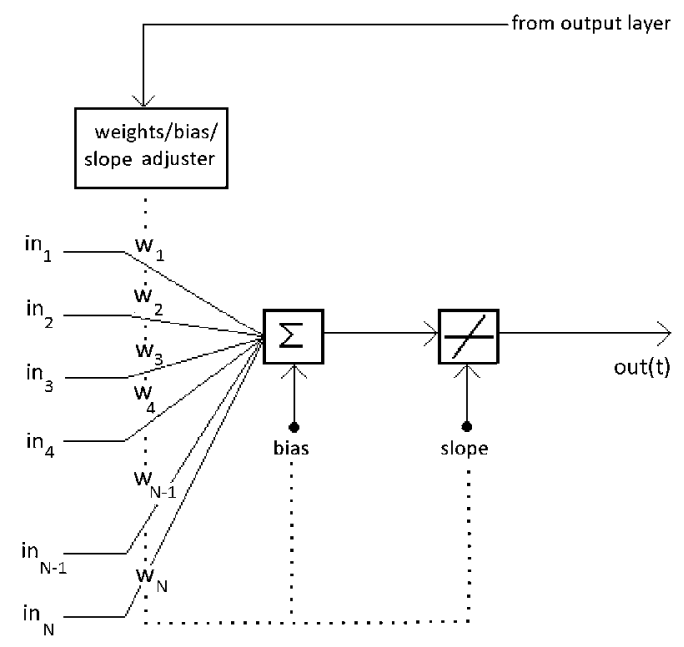

Figure 6: Scheme of a single neuron of the hidden layer of the considered ANN.

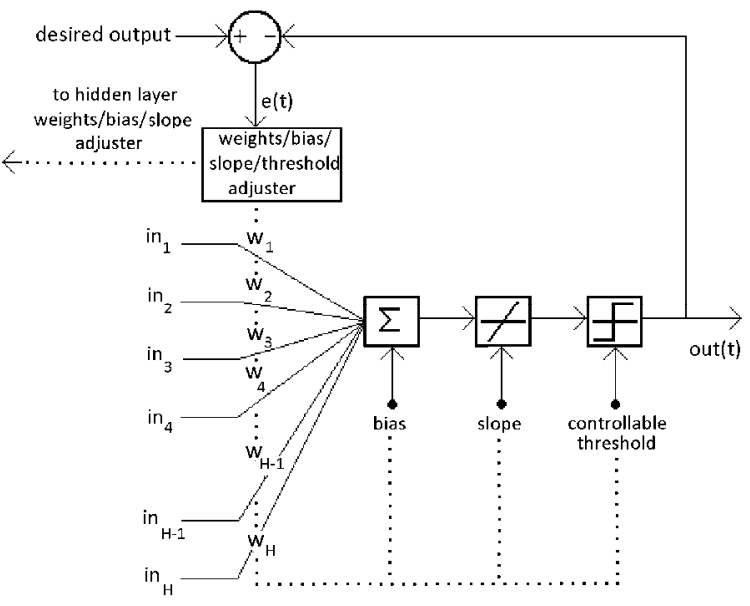

Figure 7: Scheme of a single neuron of the output layer (classifier layer) of the considered ANN.

All the $\mathrm{N}$ outputs of the input layer represent the inputs of each of $\mathrm{H}$ neurons of the hidden layer that are fed into the hidden layer whose outputs represents the inputs of the output layer which activates a single neuron for each of the considered microclimate critical situation.

Since the H neurons of hidden layer cooperates with neurons of output layer, their number is quite critical and it is must be related to the number of the output neurons, as shown in the following, to control the classifying error.

The identification of all the critical situations must be done very carefully, according to the desired corrective actions (reduction of number of visitors, etc). The number of R neurons of the output layer of the ANN must therefore be equal to the number of microclimate critical 
situations since each of them must provide a positive output only in the presence of a combination of input parameters that can generate a microclimate critical situation.

Since an alarm which is generated by the output layer is represented by a binary value and the output function of the neuron is linear, it is necessary to introduce a threshold element which provides a binary input as a function of the input value and of the controllable threshold.

The structure of neurons of hidden layer is similar to the structure of neurons of the output layer but since they must provide output values variable in a given range, to support the ANN to learn in the best way the microclimate environment, the threshold element is not present.

Since the temporal behaviour of input parameters is not known a priori, the input layer is trained in incremental mode during the operative phase.

On the contrary, since the combinations of values of input parameters which generate critical microclimate conditions are known a priori, due to a proper preliminary analysis, the hidden layer together with the output layer are trained jointly in batch mode that is the different combinations are fed into the hidden layer of the ANN and the weights/bias/slope of the hidden layer and the weights/bias/slope/threshold of the output layer are adjusted so that only a neuron provides a binary output equal to one per critical conditions considered while all the others provide a binary output equal to zero.

In this way, the output layer of the ANN, aided by the hidden layer, acts as a classifier of the critical microclimate conditions while the first layer of the ANN acts as a forecaster. The result is that the neural network behaves as an early warning system, forecasting an alarm before that a critical microclimate situation takes place.

It is obvious that the more the number of delay elements, which is the number of past values of each input parameter, and the greater the precision of the system. In general, for microclimate applications, a good compromise results in choosing a delay time $\Delta \mathrm{T}$ variable between 1 and 10 minutes, which represents a good trade-off between velocity and precision of the system.

The forecasting capabilities of the system are mainly used to antedate a microclimate critical situation and to provide a suitable early warning message to the main IoE system and to the safety/security/ emergency personnel so that they can activate all the necessary countermeasures to reduce the effects of critical situation.

To ensure a high degree of precision, it is necessary to extend as more as possible the number of past input parameters: if this number is large enough, the forecasting is accurate but the complexity of the ANN increases. For this reason, it is necessary to find a compromise between precision of the results and complexity of the ANN. In general, for microclimate applications, a good compromise results in choosing past samples variable between 10 minutes and 180 minutes which represents a good trade-off between complexity and precision of the system. The input layer of the ANNs needs a certain training time before predicting, with a high degree of precision, the behaviour of the input parameters.

Since the standard temporal length of each microclimate parameter can be variable, there must be a proper criterion which decides the value of each parameter in the case of continuously changing of this parameter inside the minimum considered input temporal interval. For this reason, a proper mean value computation unit has been used. This unit acquires the instantaneous values of the considered parameter and provides, at the end of the period $\Delta \mathrm{T}$, the mean value of the parameter itself as input to the first layer of the ANN.

The input layer of the ANN, that represent the forecasting part of net, works in real time. It is trained in incremental mode to continue to learn during its normal working activities. After the training phase, it can perform correct forecasting of future values of input microclimate parameters as a function of past parameters with an error equal to zero. 
The output layer of the neural net, aided by the hidden layer, that is the classifier part, is trained in a batch mode, since the desired actions to control the microclimate conditions are known a priori, and the results are that this part of the ANN can classify the inputs with an error that varies according to the ratio between the number of neurons that compose the hidden layer and the number of neurons that compose the output layer. For this reason, different ANN solutions characterized by different ratio between $\mathrm{H}$ neurons of hidden layer and $\mathrm{R}$ neurons of output layer have been tested, checking the related results from the output error point of view, expressed in percentage.

During the training of the net, every $10 \%$ of the total number of inputs, all the inputs have been presented to the net to evaluate the output errors. Results of error as a function of number of inputs used as training, for different values of ratio between $\mathrm{H}$ neurons of hidden layer and $\mathrm{R}$ neurons of output layer, are shown in Fig. 8.

From Fig. 8 it is possible to see that the output error decreases anyway with the increase of number of inputs but the descent slope depends on the ratio H/R. This different behaviour is due to the learning support of hidden layer: the lower the ratio $H / R$ and the lower the number of neurons of hidden layer with respect to the number of neurons of output layer. This means that the hidden layer cannot support efficiently the output layer in learning correctly the situations to be classified and the output error does not reach a value equal to zero when all the inputs have been presented to the ANN. On the contrary, the greater the ratio $\mathrm{H} / \mathrm{R}$ and the greater the number of neurons of hidden layer with respect to the number of neurons of output layer. This means that the hidden layer can support efficiently the output layer in learning correctly the situations to be classified and the output error reaches a value equal to zero when all the inputs have been presented to the ANN but also for lesser value of number of inputs when the ratio $\mathrm{H} / \mathrm{R}$ reaches values close to 1 . This mean that when the

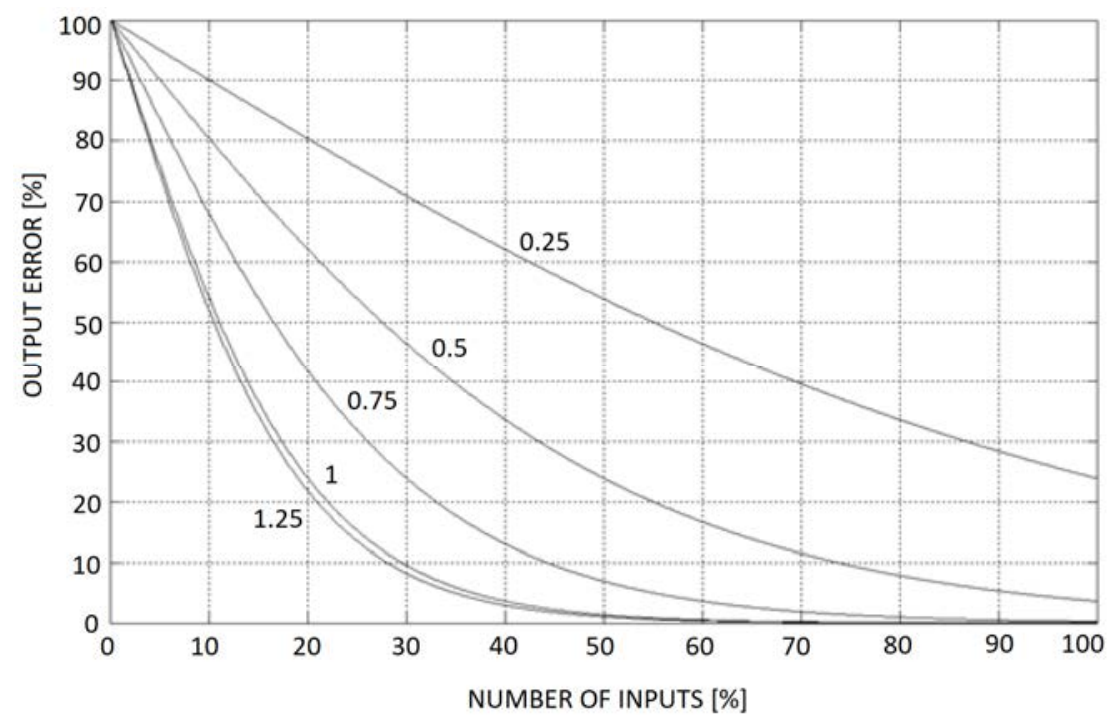

Figure 8: Output error as a function of number of inputs (both expressed in percentage), for different values of ratio between the number $\mathrm{H}$ of neurons of hidden layer and the number $\mathrm{R}$ of neurons of output layer. 
number of neurons of hidden layer tends to be equal to the number of neurons of output layer, the ANN works in a very efficient way. This performance tends to increase no more when $\mathrm{H} / \mathrm{R}$ is greater than 1 , as it is possible from Fig. 8.

The system can face also new critical situations, since it can continue to learn during its normal working activities. Therefore, after a proper training period, the ANN is capable of forecasting, with an error just equal to zero, all the critical microclimate situations that have been individuated, working as an efficient early warning system capable of anticipating the mentioned critical microclimate situations, according to the design criteria, of a reasonable time which allows to activate all the necessary countermeasures to face and control every critical situation that can verify in the Basilica.

\section{CONCLUSIONS}

The experimental microclimate monitoring system of the Papal Basilica of Saint Francis in Assisi, based on suitable microclimate monitoring modules, have been presented.

It is aimed at controlling the microclimate conditions to avoid of reaching critical conditions that could activate damaging processes of the unique frescos of the Basilica.

The system is able not only to monitor in real time the microclimate but also to forecast the future microclimate as a function of the actual situation, thanks to a proper designed artificial neural network (ANN) so that it can send this information to the IoE server which can activate all the necessary countermeasures such as reduce the number of visitors, activate proper cooling/fanning systems etc.

It represents one of the sub-systems of a proper Integrated Technological System Framework based on IoE (ITSF-IoE), that is going to be realized sub-system by subsystem, to finally support an efficient integrated multidisciplinary model for security and safety management (IMMSSM) capable of ensuring a plenty of functionalities aimed at simplifying the management of such a unique and complex site such as the considered one, not only from security and safety point of view.

\section{ACKNOWLEDGEMENTS}

The authors wish to thank Dr. Alessandro Buonpane and Mr. Andrea Buonpane for the precious support in the realization of the ad-hoc boxes of the microclimate monitoring modules (MCMM).

\section{REFERENCES}

[1] Garzia, F., Sammarco, E. \& Cusani, R., The integrated security system of the Vatican City State, International Journal of Safety \& Security Engineering, 1(1), pp. 1-17, 2011.

[2] Contardi, G., Garzia, F. \& Cusani, R., The integrated security system of the Senate of the Italian Republic, International Journal of Safety \& Security Engineering, 1(3), pp. 219-246, 2011.

[3] Garzia, F. \& Cusani, R., The integrated safety / security / communication system of the Gran Sasso mountain in Italy, International Journal of Safety \& Security Engineering, 2(1), pp. 13-39, 2012.

[4] Garzia, F. \& Cusani, R., The safety/security/communication wireless LAN of the underground Gran Sasso mountain national laboratories of the Italian Institute of Nuclear Physics, International Journal of Safety \& Security Engineering, 2(3), pp. 209-226, 2012. 
[5] Garzia, F., Sammarco, E. \& Cusani, R., Vehicle/people access control system for security management in ports, International Journal of Safety \& Security Engineering, 2(4), pp. 351-367, 2012.

[6] Garzia, F. \& Papi, L., An Internet of Everything based integrated security system for smart archaeological areas, Proceedings of the Fifty Annual IEEE International Carnahan Conference on Security Technology, pp. 64-71, 2016.

[7] Garzia, F. \& Sant'Andrea, L., The Internet of Everything based integrated security system of World War I Commemorative Museum of Fogliano Redipuglia in Italy, Proceedings of the Fifty Annual IEEE International Carnahan Conference on Security Technology, pp. 56-63, 2016.

[8] Garzia, F., An Integrated Multidisciplinary Model for Security Management Optimized Implementation Technique and Related Supporting Technological System Framework, Proceedings of the Fifty Annual IEEE International Carnahan Conference on Security Technology, pp. 107-114, 2016.

[9] Guarascio, M., Lombardi, M., Rossi, G. \& Sciarra, G., Risk analysis and acceptability criteria, WIT Transactions on the Built Environment, 94, pp.131-138, 2007.

[10] Broder, J.F. \& Tucker, E., Risk Analysis and the Security Survey, ButtherworthHeinemann, New York, 2012.

[11] Garzia, F., Handbook of Communication Security, WIT Press, 2013.

[12] Borghini, F., Garzia, F., Borghini, A. \& Borghini, G., The Psychology of Security, Emergency and Risk, WIT Press, 2016.

[13] Garzia, F., Costantino, D. \& Baiocchi, V., Security and safety management and role of laser scanning in unique and peculiar cultural heritage sites such as the Papal Basilica and the sacred convent of St. Francis in Assisi in Italy, J. of Heritage Architecture, 2(2), 2017.

[14] Angelini, M.G, Baiocchi, V., Costantino, D. \& Garzia, F., Scan to BIM for 3D Reconstruction of the Papal Basilica of Saint Francis in Assisi in Italy, The International Archives of the Photogrammetry, Remote Sensing and Spatial Information Sciences, Volume XLII-5/W1, 2017 GEOMATICS \& RESTORATION - Conservation of Cultural Heritage in the Digital Era, 22-24 May 2017, Florence, Italy, pp. 47-54. doi:10.5194/isprs-archives-XLII-5-W1-47-2017.

[15] Garzia, F. \& Cusani, R., New technique for the optimization of security communication wired networks in historical buildings, Proceedings of the Fortieth Annual IEEE International Carnahan Conference on Security Technology, pp. 116121, 2013.

[16] Garzia, F. \& Lombardi, M., The role of BIM for Safety and Security management, Int. J. Sus. Dev. Plann., 13(1), pp. 49-61, 2018.

[17] Bishop, C.M., Neural Networks for Pattern Recognition, Oxford University Press, Oxford (UK), 1995.

[18] Bishop, C.M., Pattern Recognition and Machine Learning, Oxford University Press, Oxford (UK), 2006. 